\title{
Self-organized Criticality in Spatial Evolutionary Game Theory
}

\author{
Timothy Killingback* and Michael Doebeli ${ }^{*}+$ \\ *CERN, CH-1211 Geneva 23, Switzerland; and the †Zoology Institute, University of Basel, \\ Rheinsprung 9, CH-4051 Basel, Switzerland
}

(Received on 2 September 1997, Accepted on 30 November 1997)

\begin{abstract}
Self-organized criticality is an important framework for understanding the emergence of scale-free natural phenomena. Cellular automata provide simple interesting models in which to study self-organized criticality. We consider the dynamics of a new class of cellular automata which are constructed as natural spatial extensions of evolutionary game theory. This construction yields a discrete one-parameter family of cellular automata. We show that there is a range of parameter values for which this system exhibits complex dynamics with long range correlations between states in both time and space. In this region the dynamics evolve to a self-organized critical state in which structures exist on all time and length scales, and the relevant statistical measures have power law behaviour.
\end{abstract}

(C) 1998 Academic Press Limited

\section{Introduction}

Self-organized criticality (Bak et al., 1987, 1988; Tang \& Bak, 1988; Kadanoff et al., 1989) has emerged as an important mechanism for understanding the appearance of scale-free structures in physics and a variety of other disciplines. The idea of self-organized criticality has been applied to problems in a number of fields, including geology (Bak \& Tang, 1989; Carlson \& Langer, 1989), cosmology (Chen \& Bak, 1989) and evolutionary biology (Kauffman \& Johnson, 1992; Bak \& Sneppen, 1993). Given the complexity of some of these applications it is important to have a variety of simple models in which the fundamental properties of self-organized criticality can be studied. One of the simplest and most interesting class of models in which self-organized critical behavior can occur are finite state cellular automata. Examples of such models are the cellular automaton representing a "pile of sand" (Bak et al., 1987) and the cellular automaton "Life" (Bak et al., 1989). In this paper we consider a novel class of cellular automata, whose construction is inspired by

\$Author to whom correspondence should be addressed. E-mail: doebeli@ubaclu.unibas.ch considerations from evolutionary biology. This construction yields a discrete one-parameter family of cellular automata and it is shown that self-organized criticality occurs for a whole range of parameter values in the corresponding automata. This class of cellular automata, and its natural generalizations, may provide interesting new models with which to explore self-organized critical behavior. In addition, these cellular automata represent a natural generalization of evolutionary game theory which yields interesting models of spatial evolutionary dynamics.

Evolutionary game theory (Maynard Smith \& Price, 1973; Maynard Smith, 1982) has become an important way of thinking about evolution in situations in which the fitness of particular phenotypes depends on their frequencies in the population (Maynard Smith, 1974; Parker et al., 1972; Charnov, 1982; Alexrod \& Hamilton, 1981; Axelrod, 1984; Nowak \& Sigmund, 1993). Conventionally, in evolutionary game theory, it is assumed that the population is completely homogeneous and attention is focused on the evolutionary stable strategies (ESSs), which are the fixed points of a dynamical system describing the evolutionary dynamics in strategy space. However, in many biological situ- 
ations individuals occupy well-defined territorial regions. In these circumstances they interact much more with their neighbours than with those who are far away, and their success depends to a large degree on how well they do in their interactions with their neighbours.

Such considerations lead to the concept of spatial evolutionary game theory (Nowak \& May, 1992, 1993; Nowak et al., 1994a, b; Killingback \& Doebeli, 1996). In spatial evolutionary game theory (Killingback \& Doebeli, 1996) one considers a game with a finite set of strategies $\Sigma$ and payoff matrix $E(i, j)$ for strategies $i, j \in \Sigma$, together with a spatial lattice $\Lambda$. The cells in $\Lambda$ are labeled by an index $I$, and at any point in time, each cell is occupied by a strategy from the strategy set $\Sigma$. Given any cell $I$ the set of neighboring cells to $I$ (but not including $I$ itself) is denoted by $N(I)$, and $N^{*}(I)=N(I) \cup I$ will denote the set of neighboring cells to $I$ together with $I$ itself. For a given lattice $\Lambda$ the choice of the neighborhood $N(I)$ is arbitrary and the final theory depends on this choice. However, there are usually a small number of natural choices for a minimal neighbourhood $N(I)$.

A spatial evolutionary game is defined by an association, at generation $t$, of a strategy $\sigma_{t}(I) \in \Sigma$ to each cell $I \in \Lambda$, together with a dynamical process, that is, a rule which determines the association of a strategy to each cell of $\Lambda$ at generation $t+1$. Given an initial configuration of strategies $\sigma_{0}(I)$, for all $I \in \Lambda$, this yields a recursive definition of the spatial evolutionary game once the dynamical process is specified.

The dynamical process is defined as follows. First the total score $s_{t}(I)$ of cell $I$ at generation $t$ is defined to be the sum of the payoffs resulting from playing all the neighboring cells, i.e. all the cells in $N(I)$ :

$$
s_{t}(I)=\sum_{J \in N(I)} E\left(\sigma_{t}(I), \sigma_{t}(J)\right)
$$

Using the scores $s_{t}$ we can define the dynamical process which associates a strategy to each cell of $\Lambda$ at generation $t+1$. For any $I \in \Lambda$ let $\hat{\imath} \in \Sigma$ be the strategy associated, at generation $t$, to the cell $J \in N^{*}(I)$ which has maximal score $s_{t}(J)$. Then we set $\sigma_{t+1}(I)=\hat{\imath}$; that is, the strategy at site $I$ in generation $t+1$ is that strategy from among the neighbours of $I$ and $I$ itself which gets the highest score in generation $t$. This definition corresponds to a natural evolutionary scenario in which successful phenotypes replace unsuccessful ones. Spatial evolutionary game theory, defined in this way, is a finite cellular automaton (Wolfram, 1984, 1986; Langton, 1986).

In this paper we consider a particular spatial evolutionary game: the spatial Hawk-Dove game (Killingback \& Doebeli, 1996). The spatial HawkDove game is defined to be the spatial extension of the classical Hawk-Dove game (Maynard Smith, 1982) and provides perhaps the simplest spatial model of animal conflict. Here we will show that this spatial game exhibits a remarkable variety of dynamic behaviours, including self-organized criticality.

\section{Results}

In the classical Hawk-Dove game (Maynard Smith, 1982) there are two strategies Hawk $(H)$ and Dove $(D)$ with a payoff matrix that may be written in the form $E(H, H)=1-\beta, E(H, D)=2, E(D, H)=0$, $E(D, D)=1$, where $\beta$ is a positive real parameter proportional to the cost of Hawk-Hawk encounters. We consider the spatial extension of this game as defined above. For simplicity, we will take $\Lambda$ to be a two-dimensional square lattice, and we assume that the neighborhood $N(I)$ consists of the eight cells immediately surrounding a cell $I$. To complete the definition we must specify boundary conditions: here we will work with periodic boundary conditions. Clearly this spatial Hawk-Dove game is a two-state cellular automaton.

The discrete nature of the local updating rule implies that the dynamics of the system will be unchanged if $\beta$ is varied in a sufficiently small range. However, at certain values of $\beta$ there are qualitative changes in the dynamical behaviour of the system which are associated with the success or failure of certain geometrical structures of Hawks and Doves to

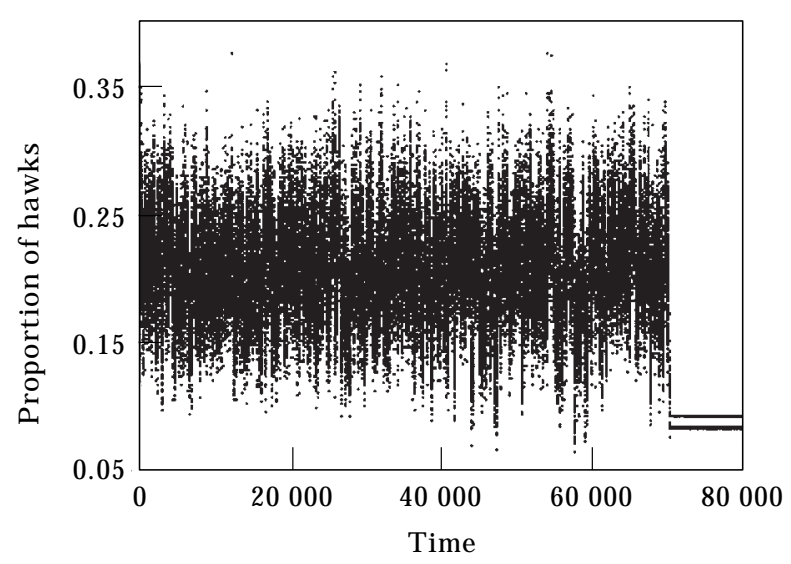

FIG. 1. A typical complex transient for $\beta=2.2$ on a $35 \times 35$ lattice starting from random initial conditions with equal amounts of Hawks and Doves. The transient lasts for approximately 70000 iterations during which the system exhibits large fluctuations in the proportion of Hawks, and after which the system relaxes to a 3-cycle. 
grow on the lattice (Killingback \& Doebeli, 1996). The spatial Hawk-Dove game therefore constitutes a discrete one-parameter family of general the global dynamics of cellular automata such as this are largely intractable to analytical investigation. However, extensive computer simulation reveals that the spatial Hawk-Dove game has a remarkably rich dynamical structure (Killingback \& Doebeli, 1996). We note first that it is possible to identify two fundamentally different dynamical regimes. In the first regime, corresponding to $\beta \in\{(1$, $9 / 7) \cup(5 / 3,2) \cup(7 / 3, \infty)\}$, the system exhibits periodic behaviour in which almost any initial configuration dynamically evolves to a cyclic state (where a fixed state is viewed as being trivially cyclic) after a relatively small number of generations. In the second regime, corresponding to $\beta \in\{(9 / 7,5 / 3) \cup(2,7 / 3)\}$, the system exhibits complicated, apparently aperiodic

(a)

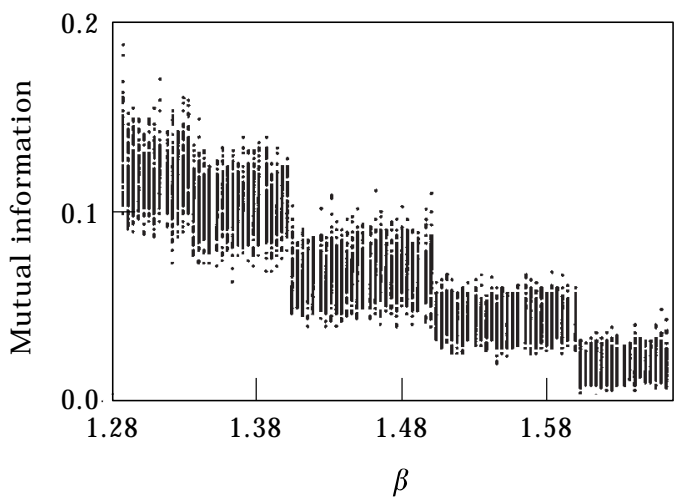

(b) two-state cellular automata, parametrized by $\beta$. In

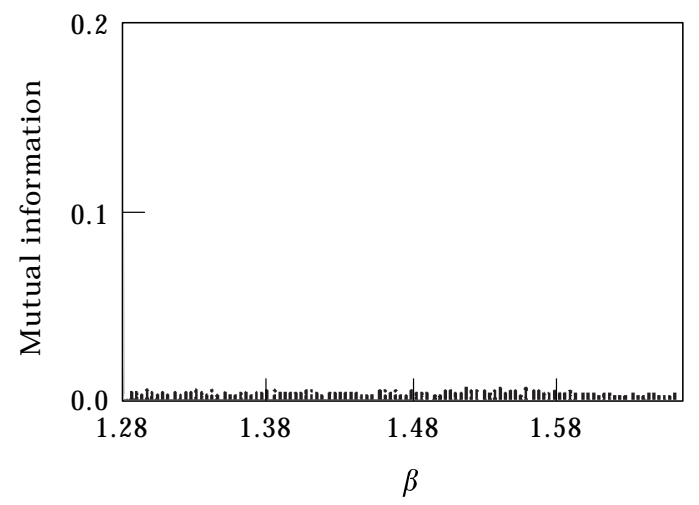

dynamical behaviour. It is this region of complicated dynamics which is of interest to us here.

We next observe that there is an important difference in the type of complicated dynamics exhibited for $\beta \in(9 / 7,5 / 3)$ and $\beta \in(2,7 / 3)$. In the latter region the system exhibits extremely long transients from almost any initial configuration before it relaxes to a simple cyclic state (Fig. 1). In addition, in this region, the length of the transients increases very rapidly (apparently at least exponentially) as the size of the lattice is increased. In the former region such extended transients are absent and the system appears to behave chaotically. The existence of very long transients suggests (Langton, 1990, 1992) that in the latter region the spatial Hawk-Dove game may exhibit complex, self-organized dynamics in which there is a significant degree of correlation between states.

A convenient quantitative measure of the degree to
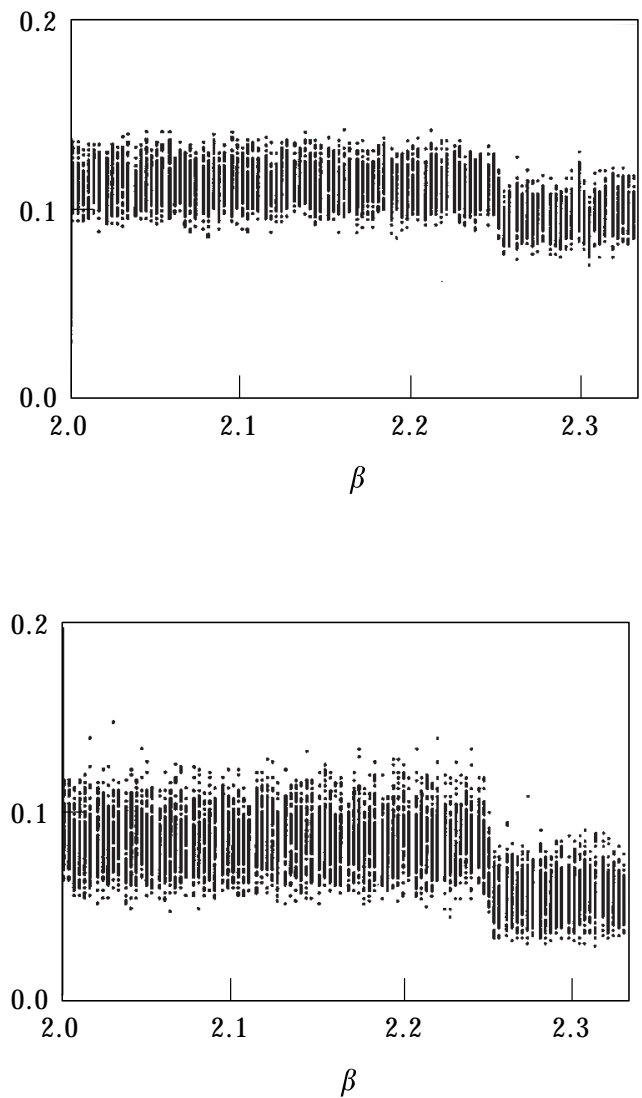

FIG. 2. Mutual information for chaotic and critical dynamics. (a) The average mutual information between a cell in one generation and the next against $\beta$ for the two regions $\beta \in[9 / 7,5 / 3]$ and $\beta \in[2,7 / 3]$. For 100 equally spaced values of $\beta$ the average mutual information is shown in 100 successive generations on a $80 \times 80$ lattice. The values of the mutual information in the two regions are roughly comparable; (b) the same as (a) but showing the average mutual information between a cell in one generation and the next-but-ninth generation. The difference between the two regions of $\beta$ values is now apparent. This suggests that the dynamics in the first region are chaotic with little temporal correlation between states. The raised value of the mutual information in the second region implies that there is a higher degree of temporal correlation between the states, which is consistent with critical dynamics. 
which the states of a cellular automaton are correlated is the mutual information (Langton, 1990, 1992; Li et al., 1990). In general, the mutual information measures the correlation between two events. It is zero if and only if the events are uncorrelated. Given two probability distributions $\left\{p_{i}\right\}$ and $\left\{p_{j}\right\}$, for the events $i$ and $j$, respectively, and the joint probability $\left\{p_{i j}\right\}$ for both event $i$ in the first variable and $j$ in the second variable to occur, the mutual information is defined by

$$
I=\sum_{i, j} p_{i j} \log _{2} \frac{p_{i j}}{p_{i} p_{j}}
$$

The average mutual information in the spatial Hawk-Dove game between a cell and itself in a subsequent generation is shown against $\beta$ in Fig. 2. The raised value of the mutual information for $\beta \in(2$, $7 / 3$ ) indicates that in this region the cells exhibit substantial temporal correlation in their behaviour. This fact, taken in conjunction with the existence of very long transients, is evidence of a critical phase transition in this region (Langton, 1990, 1992). In contrast, for $\beta \in(9 / 7,5 / 3)$ the mutual information decreases rapidly to zero as the generational gap over which it is calculated increases, suggesting that in this region the system is chaotic with little temporal correlation between states. The existence of complex dynamics would suggest that for $\beta \in(2,7 / 3)$ the spatial Hawk-Dove game is a class IV cellular automaton (Wolfram, 1984, 1986; Langton, 1986, 1990, 1992).

It is possible to obtain a more precise understanding of the suspected critical dynamics by considering the power spectrum (i.e. the absolute value squared of the Fourier transform, see Hastings \& Sugihara, 1995) of the time series given by the proportion of Hawks in each generation. The distribution of power $P(f)$ against frequency $f$, for $\beta=2.13$ and $\beta=1.35$, is shown in Fig. 3 on logarithmic scales. For $\beta=2.13$ the distribution appears to be a power law, $P(f) \propto f^{-\gamma}$, where $\gamma=0.8$. We find such power law behaviour of $P(f)$ for all $\beta \in(2,7 / 3)$ that we have studied. The occurrence of these power laws indicates that there is no natural time scale in the systems for $\beta \in(2,7 / 3)$ and is characteristic of self-organized criticality. The power law behaviour of $P(f)$ obtained for $\beta \in(2,7 / 3)$ is in marked contrast to the power spectrum obtained for $\beta \in(9 / 7,5 / 3)$, which is shown for $\beta=1.35$ in Fig. 3(b). The spectra found for $\beta \in(9 / 7,5 / 3)$ do not exhibit power law behaviour and are consistent with chaotic dynamics.

As an aside, we note that the power laws governing the time series spectra can be used to distinguish two qualitatively different dynamic regimes within the critical region $(2,7 / 3)$ itself. Already in Fig. 2 it is apparent that the mutual information changes qualitatively within the critical region at a value of $\beta \simeq 2.25$. This can be made more precise using the power spectra: for all values of $\beta \in(2,7 / 3)$ that we have studied, the exponent $\gamma$ describing the power law of the spectrum of the corresponding time series was very close to 0.8 when $\beta$ was in the interval $(2,2.25)$ [Fig. 3(a)], whereas the exponent was very close to 0.74 when $\beta$ was in the interval $(2.25,7 / 3)$. Thus the critical region seems to be subdivided into two regions that are characterized by different power law

(a)

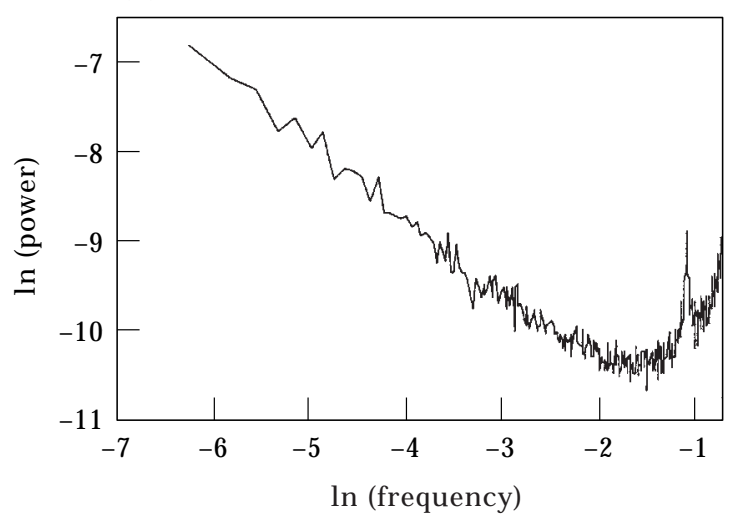

(b)

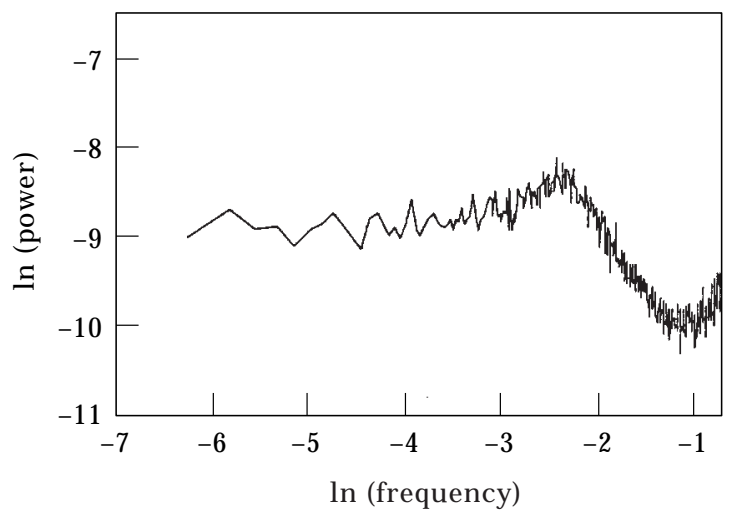

FIG. 3. (a) Power spectrum $\ln [P(f]$ of the time series given by the proportion of Hawks in each generation of the spatial Hawk-Dove game for $\beta=2.13$. The spectrum obeys a power law $P(f) \propto f^{-\gamma}$ where $\gamma \simeq 0.8$. For values of $\beta$ in the critical region the power spectrum was calculated for transient time series (i.e. before the system relaxes into a periodic state, cf. Fig. 1). The figures show the average of Fast Fourier transforms of 30 sets of 1024 data points taken from a time series on a $600 \times 600$ lattice (the lattice size was chosen so that transients become very long). The increase in power at high frequencies is a finite size effect. The discrete nature of the system implies that even a small number of changes from Hawk to Dove or vice versa from one generation to the next will, on a finite lattice, always contribute a certain minimal power at high frequency; (b) The power spectrum for $\beta=1.35$. In this case there is no power law behaviour. Instead there is a dominant range of intermediate frequencies explaining most of the variation in the time series. Such spectra are consistent with chaotic dynamics. 


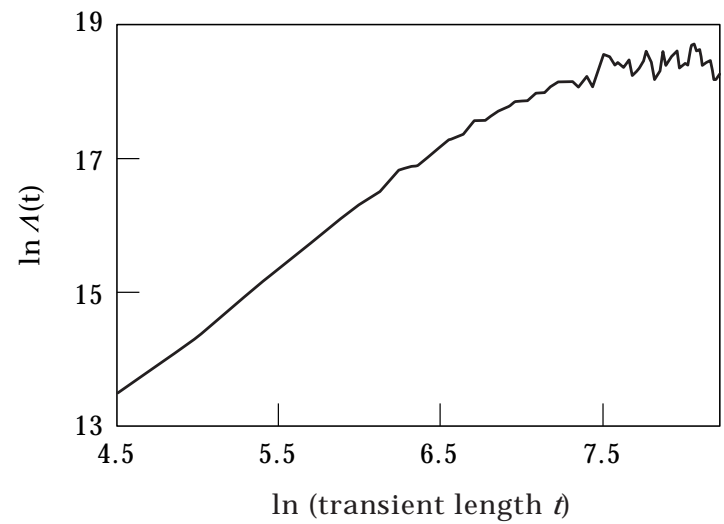

FIG. 4. Distribution of the weighted duration of avalanches $\ln [\lambda(t]$ for $\beta=2.13$. The distribution is governed by a power law $\Lambda(t) \propto t^{\mu}$, where $\mu \simeq 1.8$.

exponents. The exponent seems to be constant as a function of $\beta$ within each region, with a discontinuity at $\beta \simeq 2.25$.

We also study the following process which is related to the methods used to study the criticality of the cellular automaton "Life" (Bak et al., 1989). Starting from a random initial configuration we allow the system to evolve in time until a cyclic state is reached. We then apply a perturbation to the system at a randomly chosen cell (the perturbation consists of changing the state of a selected cell to Hawk if the cell was originally Dove or vice versa) and again allow the system to evolve to a cyclic state. By repeating this procedure a large number of times one obtains a statistical distribution for the number of "avalanches" of different durations following each perturbation. In addition we measure the size (defined as the total number of changes from Hawk to Dove and from Dove to Hawk) of the avalanches following each perturbation. We now consider the weighted duration of avalanches defined by (Christensen et al., 1991, 1992):

$$
\Lambda(t)=\sum_{s} s^{2} P(s, t)
$$

where $P(s, t)$ is the probability that an avalanche of size $s$ has duration $t$. The distribution of $\Lambda(t)$ against $t$ is shown in Fig. 4 for $\beta=2.05$ on logarithimic scales. The distribution of the weighted duration of avalanches seems to be a power law, $\Lambda(t) \propto t^{\mu}$, where $\mu=1.8$. Again we find such power law behaviour for $\Lambda(t)$ for all $\beta \in(2,7 / 3)$ that we have studied. The appearance of these power laws is a further indication that, in the region $\beta \in(2,7 / 3)$, structures occur on all time-scales. In this region, the dynamics of the spatial Hawk-Dove game naturally evolve towards a critical state, and perturbations of this critical state lead to avalanches on all time scales. Hence the system appears to be operating at a self-organized critical state. This result is particularly interesting in view of other work suggesting that certain coevolutionary systems also approach a self-organized critical state (Kauffman \& Johnson, 1992; Bak \& Sneppen, 1993).

Finally, we consider the spatial distribution of states which occur during the time evolution of the spatial Hawk-Dove game. The number of spatial Hawk clusters of size $a$ is denoted by $N(a)$ (a cluster is a configuration in which every Hawk has at least one neighbour which is also a Hawk). The distribution of $N(a)$ for $\beta=2.2$ and $\beta=1.35$ is shown in Fig. 5. For $\beta=2.2$ the distribution of $N(a)$ is close
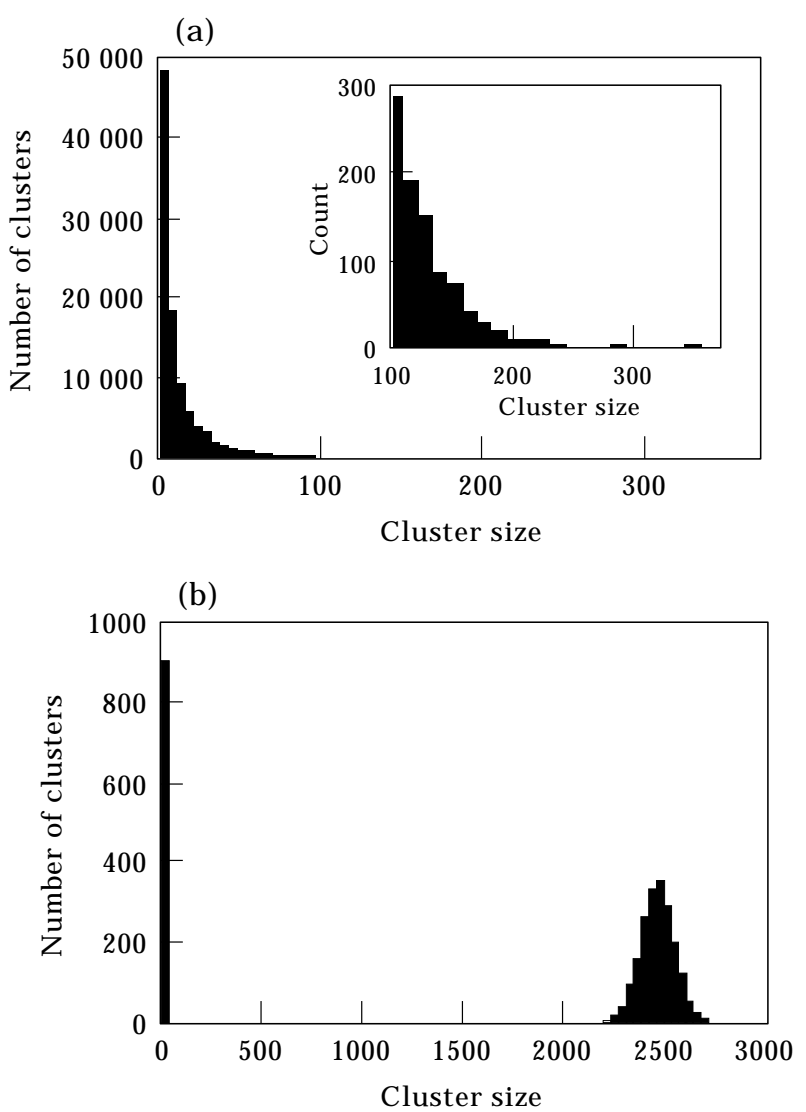

FIG. 5. Number $N(a)$ of spatial Hawk clusters of size $a$, for two values of $\beta$. (a) $\beta=2.05$. The distribution of $N(a)$ is close to a power law-although there are deviations from an exact power law, clusters occur over a wide range of size scales; (b) $\beta=1.35$. The distribution is now bimodal with many very small clusters and some very large clusters. The large number of small clusters is a consequence of the correlation length being small. The very large clusters result from the fact that in the chaotic phase the correlation strength between states is sufficiently weak that the system behaves to some degree like a random system with a given site occupation probability, which is in this case above the percolation threshold. As a result, there is one very large cluster in each generation of the spatial game. 
to a power law. Although there are deviations from an exact power law, clusters occur on a wide range of size scales. We find similar behaviour for $N(a)$ for all $\beta \in(2,7 / 3)$ that we have studied. This suggests that, for $\beta \in(2,7 / 3)$, the correlation length of the system is large, which is consistent with the existing critical dynamics in this region. This is in clear contrast with the distribution of $N(a)$ for $\beta \in(9 / 7,5 / 3)$, an example of which is shown in Fig. 5(b). Here the correlation length is small and is consistent with chaotic dynamics.

\section{Conclusions}

Using various statistical measures for dynamic processes, we have shown that there is strong evidence of a phase of self-organized criticality in the spatial Hawk-Dove game. For parameter values in this phase, the spatial Hawk-Dove game exhibits high temporal and spatial correlations between states at different lattice sites, and various processes are governed by power laws. In particular, in this phase the system exhibits extremely long transients, and the Fourier spectrum of the time series of the proportion of Hawks in the population is given by a power law. These phenomena occur for a continuous range of parameters in the spatial Hawk-Dove game.

However, there are other spatial evolutionary games which do not exhibit complex critical dynamics of this type. An interesting example of such a theory is the spatial Prisoner's Dilemma (Nowak \& May, 1992, 1993; Nowak et al., 1994a, b). The techniques used in this paper show that the dynamics of the spatial dilemma have only periodic and chaotic dynamics. Nevertheless, we conjecture that any spatial theory constructed from a classical game which possesses a mixed ESS (such as the HawkDove game, but in contrast to the Prisoner's Dilemma game, where the only ESS is the pure strategy Defect) exhibits self-organized criticality for a suitable range of parameters. As a consequence, we believe that spatial evolutionary game theory may prove to be very useful for the study of self-organized criticality in general.

The occurrence of critical dynamics in spatial evolutionary game theory raises an interesting question concerning the ubiquity of critical dynamics in spatially structured population models in general. It is known that very long transients occur in certain spatially structured ecological models of the type considered by Hastings \& Higgins (1994), and it appears that these models can exhibit critical dynamics for a suitable range of parameter values. The possibility of critical dynamics existing in a large class of spatially extended population models is significant, as the natural occurrence of $1 / f$ power spectra in critical dynamics could provide a fundamentally new mechanism to account for the increase in population variability with census interval that has been found for many natural populations.

\section{REFERENCES}

Axelrod, R. (1984). The Evolution of Cooperation. New York: Basic Books.

Axelrod, R. \& Hamilton, W. D. (1981). Science 211, 1390-1396. BAK, P. \& TANG, C. (1989). J. Geophys. Res. 95, 15635-15637.

BaK, P., Chen, K. \& Creutz, M. (1989). Nature 342, 780-782.

Bak, P. \& Sneppen, K. (1993). Phys. Rev. Lett. 71, 4083-4086.

Bak, P., Tang, C. \& Wiesenfeld, K. (1987). Phys. Rev. Lett. 59, 381-384.

Bak, P., Tang, C. \& Wiesenfeld, K. (1988). Phys. Rev. A38, 364-374.

Carlson, J. M. \& Langer, J. S. (1989). Phys. Rev. Lett. 62, 2632-2635.

Charnov, R. L. (1982). The Theory of Sex Allocation. Princeton: Princeton University Press.

Chen, K. \& BAK, P. (1989). Phys. Lett. A140, 299-302.

Christensen, K., Jensen, H. \& Fogedby, H. (1991). J. Stat. Phys. 63, 653-681.

Christensen, K., Olami, Z. \& Bak, P. (1992). Phys Rev. Lett. 68, 2417-2420.

Hastings, H. M. \& Higgins (1994).

Hastings, H. M. \& Sugihara, G. (1995). Fractals: a User's Guide for the Natural Sciences. Oxford: Oxford University Press.

Kadanoff, L. P., Nagel, S. R., Wu, L. \& Zhou, S. (1989). Phys. Rev. A39, 6524-6537.

Kauffman, S. \& Johnson, S. (1992). In: Artificial Life II (Langton, C. G., Taylor, C., Farmer, J. D. \& Rasmussen, S. eds), pp. 325-369. Reading, MA: Addison Wesley.

Killingback, T. \& Doebeli, M. (1996). Proc. R. Soc. Lond. B. 263, 1135-1144.

Langton, C. G. (1986). Physica D 22, 120-149.

Langton, C. G. (1990). Physica D 42, 12-37.

Langton, C. G. (1992). In: Artificial Life II (Langton, C. G., Taylor, C., Farmer, J. D. \& Rasmussen, S., eds), pp. 41-91. Reading, MA: Addison Wesley.

Li, W., Packard, N. H. \& Langton, C. G. (1990). Physica D 45, 77-94.

Maynard Smith, J. (1974). J. Theor. Biol. 47, 209-221.

MaYnARD SMith, J. (1982). Evolution and the Theory of Games. Cambridge: Cambridge University Press.

Maynard Smith, J. \& Price, G. R. (1973). Nature 246, 15-16.

NowaK, M. A. \& May, R. M. (1992). Nature 359, 836-829.

NowaK, M. A. \& May, R. M. (1992). Int. J. Bifurcation Chaos 3, 35-78.

NowaK, M. A. \& Sigmund, K. (1993). Nature 364, 56-58.

Nowak, M. A., Bonhoeffer, S. \& May, R. M. (1994a). Proc. Nat. Acad. Sci. USA 91, 4877-4881.

NowaK, M. A., Bonhoeffer, S. \& May, R. M. (1994b). Int. J. Bifurcation Chaos 4, 33-56.

Parker, G. A., Baker, R. R. \& Smith, V. G. F. (1972). J. Theor. Biol. 36, 529-553.

TAng, C. \& BaK, P. (1988). Phys. Rev. Lett. 60, 2347-2350.

Wolfram, S. (1984). Nature 311, 419'424.

Wolfram, S. (ed.) (1986). Theory and Applications of Cellular Automata. Singapore: World Scientific. 